\title{
Chaos Expansion of Local Time of Fractional Brownian Motions
}

\author{
Yaozhong $\mathrm{Hu}^{1)}$ and Bernt $\varnothing \mathrm{ksendal}{ }^{2), 3)}$
}

March 29, 2005

1) Department of Mathematics, University of Kansas 405 Snow Hall, Lawrence, Kansas 66045-2142, USA.

Email: hu@math.ukans.edu

2) Department of Mathematics, University of Oslo Box 1053 Blindern, N-0316 Oslo, Norway.

Email: oksendal@math.uio.no

3) Norwegian School of Economics and Business Administration, Helleveien 30, N-5045 Bergen, Norway.

\begin{abstract}
We find the chaos expansion of local time $\ell_{T}^{(H)}(x, \cdot)$ of fractional Brownian motion with Hurst coefficient $H \in(0,1)$ at a point $x \in \mathbb{R}^{d}$. As an application we show that when $H_{0} d<1$ then $\ell_{T}^{(H)}(x, \cdot) \in L^{2}(\mu)$. Here $\mu$ denotes the probability law of $B^{(H)}$ and $H_{0}=\max \left\{H_{1}, \cdots, H_{d}\right\}$. In particular, we show that when $d=1$ then $\ell_{T}^{(H)}(x, \cdot) \in L^{2}(\mu)$ for all $H \in(0,1)$.
\end{abstract}

\section{Introduction}

Let $H=\left(H_{1}, H_{2}, \ldots, H_{d}\right)$ with $0<H_{j}<1, j=1,2, \ldots, d$. Let $B^{(H)}(t)=$ $\left(B_{1}^{(H)}(t), \ldots, B_{d}^{(H)}(t)\right), t \in \mathbb{R}$, be a d-dimensional fractional Brownian motion, i.e. $B^{(H)}(t)$ is a Gaussian process in $\mathbb{R}^{d}$ such that

$$
B^{(H)}(0)=0=\mathbb{E}\left[B^{(H)}(t)\right]
$$

for all $t \in \mathbb{R}$ and

$$
\mathbb{E}\left(B_{j}^{(H)}(t) B_{k}^{(H)}(s)\right)=\frac{1}{2}\left\{|s|^{2 H_{j}}+|t|^{2 H_{j}}-|s-t|^{2 H_{j}}\right\} \delta_{j k}, \quad 1 \leq j, k \leq d
$$

AMS 1991 subject classifications. Primary 60H05, 60H10;

Key words and phrases: Fractional Brownian motions; Local time; Chaos expansion; Asymptotic behavior. 
where

$$
\delta_{j k}= \begin{cases}0 & \text { when } j \neq k \\ 1 & \text { when } j=k\end{cases}
$$

Here $\mathbb{E}=\mathbb{E}_{\mu}$ denotes the expectation with respect to the probability law $\mu=\mu_{H}$ for $B^{(H)}(\cdot)$. This means that we are considering $d$ independent fractional Brownian motions with Hurst parameters $H_{1}, H_{2}, \ldots, H_{d}$ respectively.

In this paper we study the local time of fractional Brownian motion $B^{(H)}(t)$, heuristically described by:

$$
\ell_{T}(x)=\ell_{T}^{(H)}(x)=\int_{0}^{T} \delta\left(B^{H}(t)-x\right) d t,
$$

where $\delta$ is the Dirac delta function, which is approximated by

$$
P_{\varepsilon}(x)=\frac{1}{(2 \pi \varepsilon)^{d / 2}} e^{-\frac{|x|^{2}}{2 \varepsilon}}=\frac{1}{(2 \pi)^{d}} \int_{\mathbb{R}^{d}} e^{i x \xi-\frac{1}{2} \varepsilon \xi^{2}} d \xi, \quad x \in \mathbb{R}^{d}
$$

where $i=\sqrt{-1}$. This means that formally

$$
\delta(x)=\lim _{\varepsilon \rightarrow 0} P_{\varepsilon}(x)=\frac{1}{(2 \pi)^{d}} \int_{\mathbb{R}^{d}} e^{i x \xi} d \xi
$$

We shall use the above formal expression for $\delta$. The justification of this is similar to those in $[\mathrm{AHZ}],[\mathrm{Hu} 1]$ etc. Below we will give another rigorous justification based on fractional white noise analysis.

The purpose of this paper is to find the chaos expansion of local time $\ell_{T}^{(H)}(x)$ of fractional Brownian motion at a point $x \in \mathbb{R}^{d}$ (see Theorem 5.1). As an application of this we show that when $H_{0} d<1$ then $\ell_{T}^{(H)}(x, \cdot) \in L^{2}(\mu)$ (see Theorem 5.3). Here $\mu$ denotes the probability law of $B^{(H)}$ and $H_{0}=\max \left\{H_{1}, \cdots, H_{d}\right\}$. In particular, we show that when $d=1$ then $\ell_{T}^{(H)}(x, \cdot) \in L^{2}(\mu)$ for all $H \in(0,1)$.

If we compare with the standard Brownian motion case with $H_{j}=\frac{1}{2}$ for all $j=1,2, \ldots, d$, Theorem 5.3 is sharp, because in this case it is well-known that the local time exists as an element of $L^{2}(\mu)$ only for $d=1$.

\section{Fractional Calculus and FBM}

First let us recall some results from $[\mathrm{Hu} 4]$ and $[\mathrm{SKM}]$. We refer to the above mentioned references for more details.

Fractional integrals of order $\alpha \in(0,1)$ (of Riemann-Liouville type) of a function $f$ on $\mathbb{R}$ are defined as

$$
I_{ \pm}^{\alpha} f(x)=\frac{1}{\Gamma(\alpha)} \int_{0}^{\infty} t^{\alpha-1} f(x \pm t) d t
$$


where $\Gamma(\alpha)$ is the gamma function of $\alpha$. They are also called fractional integral of Weyl type in $[\mathrm{MvN}]$. When $\alpha=-\beta \in(-1,0)$ is negative $I_{ \pm}^{\alpha}$ will be the fractional derivatives (of Marchaud type):

$$
I_{ \pm}^{\alpha} f(x)=\mathrm{D}_{ \pm}^{\beta} f(x)=\frac{\beta}{\Gamma(1-\beta)} \int_{0}^{\infty} \frac{f(x)-f(x \mp t)}{t^{1+\beta}} d t .
$$

Denote the Fourier transform of a function $f$ by

$$
\hat{f}(\xi)=\mathcal{F}(f)(\xi)=\int_{\mathbb{R}} e^{i x \xi} f(x) d x, \quad \xi \in \mathbb{R} .
$$

The Fourier transform of $I_{ \pm}^{\alpha} f$ is given by

$$
\mathcal{F}\left(I_{ \pm}^{\alpha} f\right)(\xi)=(i \xi)^{\mp \alpha} \hat{f}(\xi), \quad \xi \in \mathbb{R},
$$

where

$$
(i \xi)^{-\alpha}=|\xi|^{-\alpha} e^{\mp \frac{\alpha \pi i}{2} \operatorname{sign}(\xi)} .
$$

The following equation holds

$$
\int_{\mathbb{R}} g(x) I_{ \pm}^{\alpha} f(x) d x=\int_{\mathbb{R}} f(x) I_{\mp}^{\alpha} g(x) d x .
$$

First let us define stochastic integral for one fractional Brownian motion (FBM) of arbitrary Hurst parameter $H \in(0,1)$.

Assume that $B^{(H)}=\left(B^{(H)}(t), t \in \mathbb{R}\right)$ is an FBM with Hurst parameter $H \in(0,1)$. It is well-known that $B^{(H)}$ is also given by

$$
\begin{aligned}
B^{(H)}(t)= & \frac{c_{H}}{\Gamma(H+1 / 2)}\left\{\int_{-\infty}^{0}\left[(t-s)^{H-1 / 2}-(-s)^{H-1 / 2}\right] d B(s)\right. \\
& \left.+\int_{0}^{t}(t-s)^{H-1 / 2} d B(s)\right\}, \quad t \in \mathbb{R},
\end{aligned}
$$

where $B=(B(s), s \in \mathbb{R})$ is a Wiener process on some probability space $(\Omega, \mathcal{F}, P)$ and

$$
c_{H}=\frac{\pi H(1-2 H)}{\Gamma(2-2 H) \cos (\pi H)} .
$$

Using the notation of fractional calculus and white noise calculus, we may write

$$
\begin{aligned}
B^{(H)}(t) & =\frac{c_{H}}{\Gamma(H+1 / 2)}\left[\int_{-\infty}^{t}(t-s)^{H-1 / 2} \dot{B}(s) d s-\int_{-\infty}^{0}(-s)^{H-1 / 2} \dot{B}(s) d s\right] \\
& =c_{H}\left[I_{+}^{H+1 / 2}(\dot{B})(t)-I_{+}^{H+1 / 2}(\dot{B})(0)\right]
\end{aligned}
$$


where $\dot{B}(s)=\frac{d B(s)}{d s}$ is white noise. Thus

$$
\begin{aligned}
\dot{B}^{(H)}(t) & :=\frac{d}{d t} B^{(H)}(t) \\
& =c_{H} \frac{d}{d t} I_{+}^{H+1 / 2}(\dot{B})(t) \\
& =c_{H} I_{+}^{H-1 / 2}(\dot{B})(t),
\end{aligned}
$$

using that $\frac{d}{d t} I_{+}^{\alpha} f(t)=I_{+}^{\alpha-1} f(t)$. Thus we see that $\dot{B}^{(H)}$ is the fractional integral of order $H-1 / 2$ of the white noise $\dot{B}$ when $H>1 / 2$ and $\dot{B}^{(H)}$ is the fractional derivative of order $1 / 2-H$ of the white noise $\dot{B}$ when $H<1 / 2$ up to a constant multiple.

Using the adjoint operator of $I_{+}^{H-1 / 2}$ and (2.6), we may write for nice (deterministic) functions $f$

$$
\begin{aligned}
\int_{\mathbb{R}} f(t) d B^{(H)}(t) & =\int_{\mathbb{R}} f(t) \dot{B}^{(H)}(t) d t=c_{H} \int_{\mathbb{R}} f(t) I_{+}^{H-1 / 2}(\dot{B})(t) d t \\
& =c_{H} \int_{\mathbb{R}} I_{-}^{H-1 / 2}(f)(t) \dot{B}(t) d t \\
& =c_{H} \int_{\mathbb{R}} I_{-}^{H-1 / 2}(f)(t) d B(t) .
\end{aligned}
$$

This identity motivates our definition of stochastic integral, and we shall use this as our definition of stochastic integral for FBM (of any Hurst parameter $H \in(0,1)$ ).

For all $\alpha \in \mathbb{R}$, introduce the following Hilbert space

$$
\begin{aligned}
L_{\alpha}^{2}(\mathbb{R}) & =\left\{f: \mathbb{R} \rightarrow \mathbb{R} ;|\xi|^{\alpha} \hat{f}(\xi) \in L^{2}(\mathbb{R})\right\} \\
& =\left\{f: \mathbb{R} \rightarrow \mathbb{R} ; \mathcal{F}\left(I_{-}^{\alpha} f\right) \in \mathrm{L}^{2}(\mathbb{R})\right\} \\
& =\left\{f: \mathbb{R} \rightarrow \mathbb{R} ; \mathcal{F}\left(I_{+}^{\alpha} f\right) \in \mathrm{L}^{2}(\mathbb{R})\right\}
\end{aligned}
$$

with the Hilbert norm

$$
\|f\|_{\alpha}^{2}:=\frac{c_{H}^{2}}{2 \pi} \int_{\mathbb{R}}|\xi|^{2 \alpha}|\hat{f}(\xi)|^{2} d \xi
$$

Denote the Hilbert space

$$
\Theta_{H}:=L_{H-1 / 2}^{2}(\mathbb{R}) \text {. }
$$

It is easy to see that a function $f$ is such that $I_{-}^{H-1 / 2} f$ is well-defined as an element in $L^{2}(\mathbb{R})$ if and only if $f \in \Theta_{H}$ and moreover,

$$
\left\langle I_{-}^{H-1 / 2} f, I_{-}^{H-1 / 2} g\right\rangle_{L^{2}(\mathbb{R})}=\frac{1}{c_{H}^{2}}\langle f, g\rangle_{\Theta_{H}}, \quad \forall f, g \in \Theta_{H} .
$$

Now we may define 
Definition 2.1 Let $f \in \Theta_{H}$. Then we say that $f$ is integrable with respect to $B^{(H)}$ and we define

$$
\int_{\mathbb{R}} f(t) d B^{(H)}(t)=c_{H} \int_{\mathbb{R}} I_{-}^{H-1 / 2}(f)(t) d B(t) .
$$

The moments of $\int_{\mathbb{R}} f(t) d B^{(H)}(t)$ are computed as follows. For the first moment it is easy to see that

$$
\mathbb{E}\left(\int_{\mathbb{R}} f(t) d B^{(H)}(t)\right)=0 .
$$

The second moment is

$$
\mathbb{E}\left(\int_{\mathbb{R}} f(t) d B^{(H)}(t) \int_{\mathbb{R}} g(t) d B^{(H)}(t)\right)=\langle f, g\rangle_{\Theta_{H}} .
$$

Since $\int_{\mathbb{R}} f(t) d B^{(H)}(t)$ is a Gaussian random variable, we have

$$
\mathbb{E}\left[\left(\int_{\mathbb{R}} f(t) d B^{(H)}(t)\right)^{n}\right]= \begin{cases}0 & \text { when } n \text { is an odd positive integer } \\ \frac{n !}{2^{n / 2}(n / 2) !}\|f\|_{\Theta_{H}}^{n} & \text { when } n \text { is an even positive integer }\end{cases}
$$

To see that our definition coincides with the conventional stochastic integral defined by the limit Riemannian sums, we cite

Proposition 2.2 Let $0<a<b<\infty$ and let $f(t)=\chi_{[a, b]}(t)$ be the indicator function of the interval $[a, b]$. Then $I_{-}^{\alpha}(f)$ is well-defined and $I_{-}^{\alpha}(f) \in L^{2}(\mathbb{R})$. Moreover,

$$
\int_{\mathbb{R}_{+}} f(t) d B^{(H)}(t)=B^{(H)}(b)-B^{(H)}(a) .
$$

Proof See [Hu4].

From this proposition, we see by linearity that the simple (step) functions are integrable and their stochastic integral coincides with the usual expression:

Corollary 2.3 Let $f(t)=\sum_{j=1}^{N-1} c_{j} \chi_{\left[t_{j}, t_{j+1}\right)(t)}$ be a step function, where $t_{1}<t_{2}<\cdots<t_{N}$ and $c_{j} \in \mathbb{R}$. Then $f$ is integrable with respect to $B^{(H)}(t)$ and

$$
\int_{\mathbb{R}} f(t) d B^{(H)}(t)=\sum_{j=1}^{N-1} c_{j}\left(B^{(H)}\left(t_{j+1}\right)-B^{(H)}\left(t_{j}\right)\right) .
$$

\section{Multiple stochastic integrals and Wiener chaos}

As in previous sections let $B^{(H)}=\left(B^{(H)}(t), t \in \mathbb{R}\right)$ be a 1-dimensional fractional Brownian motion on the probability space $(\Omega, \mathcal{F}, P)$. We assume that $\mathcal{F}$ is the $\sigma$-algebra generated by $B^{(H)}$. Let $\mathcal{P}$ be the set of polynomial functionals of $B^{(H)}$ on $(\Omega, \mathcal{F}, P)$ of the following form

$$
P=p\left(B^{(H)}\left(t_{1}\right), \cdots, B^{(H)}\left(t_{n}\right)\right)
$$


for some $t_{1}, \cdots, t_{n} \in \mathbb{R}$ and some polynomial $p$. Let $\mathcal{P}_{n}$ be the set of polynomials of above form such that $p$ is a polynomial of degree less than or equal to $n$. It is well-known that $\mathcal{P}$ is a dense subset of $L^{2}(\Omega, \mathcal{F}, P)$. Let $\mathcal{H}_{n}$ be the $L^{2}(\Omega, \mathcal{F}, P)$ closure of $\mathcal{P}_{n}$ and $\mathbb{H}_{n}$ be the orthogonal complement of $\mathcal{H}_{n-1}$ in $\mathcal{H}_{n}$. Then it is well-known that any element $F$ of $L^{2}(\Omega, \mathcal{F}, P)$ can be written as

$$
F=\sum_{n=0}^{\infty} F_{n}
$$

where $F_{n} \in \mathbb{H}_{n}$. This expansion relates $F$ to an element in the Fock space $\Gamma(\mathbb{H})$ over $\mathbb{H}$, which we will also use in what follows. Generally these results are called chaos expansions. We refer to $[\mathrm{Ne}]$ and $[\mathrm{J}]$ for more details. In this section we shall correspond $F_{n}$ to a multiple Itô type integral that we are going to introduce.

Define the Hermite polynomials $H_{n}(x)$ by

$$
H_{n}(x)=(-1)^{n} e^{\frac{x^{2}}{2}} \frac{d^{n}}{d x^{n}}\left(e^{-\frac{x^{2}}{2}}\right), \quad n=0,1,2, \cdots
$$

The first few Hermite polynomials are

$$
\begin{gathered}
h_{0}(x)=1, \quad h_{1}(x)=x, \quad h_{2}(x)=x^{2}-1, \\
h_{3}(x)=x^{3}-3 x, \quad h_{4}(x)=x^{4}-6 x^{2}+3, \cdots .
\end{gathered}
$$

The generating function is

$$
e^{t x-\frac{x^{2}}{2}}=\sum_{n=0}^{\infty} \frac{t^{n}}{n !} H_{n}(x), \quad \forall t, x \in \mathbb{R} .
$$

Let $\mathbb{H}=\Theta_{H}$ be the Hilbert space introduced in Section 2. The symmetric tensor product $\mathbb{H}^{\otimes n}$ of $\mathbb{H}$ can be defined in a standard way. If $f \in \mathbb{H}$, then $f^{\otimes n}$ is in $\mathbb{H}^{\otimes n}$. We define for $f \in \mathbb{H}$ with $\|f\|_{\mathbb{H}}=1$ the multiple integral $I_{n}\left(f^{\otimes n}\right)$ by the following:

$$
I_{n}\left(f^{\otimes n}\right)=H_{n}\left(\int_{\mathbb{R}} f(t) d B^{(H)}(t)\right) .
$$

When $f=\sum_{k=1}^{N} c_{k} f_{k}^{\otimes n}$, we define

$$
I_{n}(f)=\sum_{k=1}^{N} c_{k} I_{n}\left(f_{k}^{\otimes n}\right) .
$$

We can then define $I_{n}\left(f_{1} \otimes f_{2} \otimes \cdots \otimes f_{n}\right)$ by polarization. Finally a limiting argument enables us to define $I_{n}(f)$ for all $f \in \mathbb{H}^{\otimes n}$ and moreover, we have

$$
\mathbb{E}\left(I_{n}(f)\right)^{2}=n !\|f\|_{\mathbb{H}^{\otimes}}^{2} .
$$

The following theorem can be shown exactly the same way as in [DHP], [Hu2], [HØ]. 
Theorem 3.1 Let $F \in L^{2}(\mu)$. Then there is $f_{n} \in \mathbb{H}^{\otimes n}, n=1,2, \cdots$ such that

$$
F=\mathbb{E}(F)+\sum_{n=1}^{\infty} \frac{1}{n !} I_{n}\left(f_{n}\right)
$$

Moreover,

$$
\mathbb{E}\left(F^{2}\right)=(\mathbb{E}(F))^{2}+\sum_{n=1}^{\infty} \frac{1}{n !}\left\|f_{n}\right\|_{\mathbb{H}^{\otimes n}}^{2}
$$

Using the Fourier transform we may identify the symmetric Hilbert space $\mathbb{H}^{\otimes n}$ as

$$
\begin{aligned}
\mathbb{H}^{\otimes n} & =\left\{f: \mathbb{R}^{n} \rightarrow \mathbb{R}, f \text { is symmetric and } \quad \mathrm{D}^{\gamma, \cdots, \gamma} f \in L^{2}\left(\mathbb{R}^{n}\right)\right\} \\
& =\left\{f: \mathbb{R}^{n} \rightarrow \mathbb{R}, f \text { is symmetric and } \int_{\mathbb{R}^{n}}\left|\xi_{1} \cdots \xi_{n}\right|^{2 \gamma}\left|\hat{f}\left(\xi_{1}, \cdots, \xi_{n}\right)\right|^{2} d \xi_{1} \cdots d \xi_{n}<\infty\right\}
\end{aligned}
$$

where $\gamma=1 / 2-H$. Denote

$$
\int_{\mathbb{R}^{n}} f\left(t_{1}, \cdots, t_{n}\right) d B_{t_{1}}^{H} \cdots d B_{t_{n}}^{H}=I_{n}(f)
$$

if $f\left(t_{1}, \cdots, t_{n}\right), t_{1}, \cdots, t_{n} \in \mathbb{R}$ is in $\mathbb{H}_{n}$. It is easy to check that

$$
\int_{\mathbb{R}^{n}} f\left(t_{1}, \cdots, t_{n}\right) d B_{t_{1}}^{H} \cdots d B_{t_{n}}^{H}=c_{H}^{n} \int_{\mathbb{R}^{n}} \mathrm{D}^{\gamma, \cdots, \gamma} f\left(t_{1}, \cdots, t_{n}\right) d B_{t_{1}} \cdots d B_{t_{n}},
$$

where the last multiple stochastic integral is standard multiple Itô integral with respect to classical Brownian motion and

$$
\begin{aligned}
& \mathbb{E}\left(\int_{\mathbb{R}^{n}} f\left(t_{1}, \cdots, t_{n}\right) d B_{t_{1}}^{H} \cdots d B_{t_{n}}^{H}\right)^{2} \\
= & c_{H}^{n} \int_{\mathbb{R}^{n}}\left|\mathrm{D}^{\gamma, \cdots, \gamma} f\left(t_{1}, \cdots, t_{n}\right)\right|^{2} d t_{1} \cdots d t_{n} \\
= & \frac{c_{H}^{n}}{(2 \pi)^{n}} \int_{\mathbb{R}^{n}}\left|\xi_{1} \cdots \xi_{n}\right|^{2 \gamma}\left|\hat{f}\left(\xi_{1}, \cdots, \xi_{n}\right)\right|^{2} d \xi_{1} \cdots d \xi_{n} .
\end{aligned}
$$

Example 3.2 Let $f \in \mathbb{H}$. Then it is well-known that $f^{\otimes n} \in \mathbb{H}_{n}$. It is easy to check that

$$
I_{n}\left(f^{\otimes n}\right)=\sum_{k \leq n / 2} \frac{(-1)^{k} n !\|f\|_{\mathbb{H}}^{2 k}}{2^{k} k !(n-2 k) !}\left(\int_{\mathbb{R}} f(t) d B^{(H)}(t)\right)^{n-2 k} .
$$

Example 3.3 Let $f \in \mathbb{H}$. Let $F(t)=f(t) I_{n}\left(f^{\otimes n}\right), t \in \mathbb{R}$. It is easy to see that

$$
\begin{aligned}
\int_{\mathbb{R}} F(t) d B^{(H)}(t) & =I_{n+1}\left(f^{\otimes(n+1)}\right) \\
& =\sum_{k \leq n / 2} \frac{(-1)^{k}(n+1) !}{2^{k} k !(n-2 k) !}\left(\int_{\mathbb{R}} f(t) d B^{(H)}(t)\right)^{n+1} .
\end{aligned}
$$




\section{Local time for fractional Brownian motion}

In this section we give an introduction to local time $\ell_{T}(x)$ of fractional Brownian motion $B^{(H)}(t)=\left(B^{\left(H_{1}\right)}(t), \ldots, B^{\left(H_{d}\right)}(t)\right)$ at points $x \in \mathbb{R}^{d}$ up to time $T>0$. Our presentation is based on the (Donsker) delta function $\delta_{B^{(H)}(t)}(x)=\delta\left(B^{(H)}(t)-x\right)$ of $B^{(H)}(t)$ at $x \in \mathbb{R}^{d}$, a concept we will make precise using the fractional white noise theory introduced in $[\mathrm{H} \varnothing]$.

We will use the results and notation from $[\mathrm{H} \varnothing]$ and $[\mathrm{H} \varnothing \mathrm{UZ}]$. For the convenience of the reader we briefly review the material we need.

Fix a $d$-dimensional Hurst parameter

$$
H=\left(H_{1}, H_{2}, \ldots, H_{d}\right) \in(0,1)^{d} .
$$

Denote $\Theta_{j}=\Theta_{H_{j}}$. Let $\mathcal{S}(\mathbb{R})$ be the Schwartz space of rapidly decreasing smooth functions on $\mathbb{R}$. The dual is the space $\mathcal{S}^{\prime}(\mathbb{R})$ of tempered distributions. For $j=1,2, \ldots, d$ let $\mu_{j}=\mu_{H_{j}}$ be the fractional white noise probability measure on $\mathcal{S}^{\prime}(\mathbb{R})$ defined by the property

$$
\int_{\mathcal{S}^{\prime}(\mathbb{R})} e^{i\langle\omega, f\rangle} d \mu_{j}(\omega)=e^{-\frac{1}{2}\|f\|_{\Theta_{j}}^{2}} \quad \text { for all } f \in \mathcal{S}(\mathbb{R}),
$$

where $\langle\omega, f\rangle$ denotes the action of $\omega \in \mathcal{S}^{\prime}(\mathbb{R})$ on $f \in \mathcal{S}(\mathbb{R})$. Then we get the isometries

$$
\|\langle\cdot, f\rangle\|_{L^{2}\left(\mu_{j}\right)}^{2}=\|f\|_{H_{j}}^{2} ; \quad j=1, \cdots, d
$$

and using this we may define, for each $t \in \mathbb{R}$,

$$
\widetilde{B}^{\left(H_{j}\right)}(t)=\widetilde{B}^{\left(H_{j}\right)}(t, \omega)=\left\langle\omega, \chi_{[0, t]}(\cdot)\right\rangle \quad \text { in } L^{2}\left(\mu_{j}\right) .
$$

where

$$
\chi_{[0, t]}(x)= \begin{cases}1 & \text { if } 0<x<t \\ -1 & \text { if } t<x \leq 0 \\ 0 & \text { otherwise }\end{cases}
$$

It is easy to check that

$$
\mathbb{E}_{\mu_{j}}\left[\widetilde{B}^{\left(H_{j}\right)}(t) \widetilde{B}^{\left(H_{j}\right)}(s)\right]=\frac{1}{2}\left\{|s|^{2 H_{j}}+|t|^{2 H_{j}}-|s-t|^{2 H_{j}}\right\}
$$

and that $\widetilde{B}^{\left(H_{j}\right)}(t)$ is a Gaussian process. Therefore $\widetilde{B}^{\left(H_{j}\right)}(t)$ has a continuous version $B^{\left(H_{j}\right)}(t)$, which by (4.4) is a fractional Brownian motion with Hurst parameter $H_{j}$. Define

$$
B^{(H)}(t):=\left(B^{\left(H_{1}\right)}(t), \ldots, B^{\left(H_{d}\right)}(t)\right) \in \mathbb{R}^{d} .
$$

Then $B^{(H)}(t)$ is a $d$-dimensional fractional Brownian motion with Hurst parameter $H=$ $\left(H_{1}, \ldots, H_{d}\right) \in(0,1)^{d}$ with respect to the probability measure $\mu=\mu^{(H)}$ defined on $\Omega:=$ $\left(\mathcal{S}^{\prime}(\mathbb{R})\right)^{d}$ by

$$
\mu=\mu_{1} \times \cdots \times \mu_{d} .
$$


This is the (version of the) fractional Brownian motion we will work with from now on.

Put

$$
\mathbb{L}_{H}^{2}(\mathbb{R})=\left\{f=\left(f_{1}, \cdots, f_{d}\right) ; f_{j} \in L_{H-1 / 2}^{2}(\mathbb{R}) \forall j\right\}
$$

(see (2.9)) The stochastic integral of $f \in \mathbb{L}_{H}^{2}(\mathbb{R})$ is defined by (see Def. 2.1)

$$
\int_{0}^{T} f(s) d B^{(H)}(s)=\sum_{j=1}^{d} f_{j}(s) d B_{j}^{(H)}(s) ; \quad f=\left(f_{1}, \ldots, f_{d}\right) \in \mathbb{L}_{H}^{2}(\mathbb{R}) .
$$

Multiple stochastic integrals and the chaos expansion etc can be extended to the $d$-dimensional fractional Brownian motion $B^{(H)}$ easily.

We can now follow the approach in $[\mathrm{H} \varnothing]$ (see also [HØZ] and [ØZ]) and define the space $(\mathcal{S})_{H} \subset L^{2}(\mu)$ of fractional stochastic test functions and the space $(\mathcal{S})_{H}^{*} \supset L^{2}(\mu)$ of fractional stochastic (Hida) distributions. We can also define the Wick product $\diamond:(\mathcal{S})_{H}^{*} \times(\mathcal{S})_{H}^{*} \rightarrow$ $(\mathcal{S})_{H}^{*}$. As before the Wick product is a commutative and associative binary operation on $(\mathcal{S})_{H}^{*} \times(\mathcal{S})_{H}^{*}$, distributive over addition. We refer to [HØUZ] for the general theory of white noise in the standard case. Most of the theory carries over to the fractional case and will be used without further proofs.

We now proceed as in $[\mathrm{A} \varnothing \mathrm{U}]$ and define the Donsker delta function as follows:

Definition 4.1 Let $Y: \Omega \rightarrow \mathbb{R}^{d}$ be a random variable which also belongs to $(\mathcal{S})_{H}^{*}$. Then a continuous function

$$
\delta_{Y}(\cdot): \mathbb{R}^{d} \rightarrow(\mathcal{S})_{H}^{*}
$$

is called a Donsker delta function of $Y$ if it has the property that

$$
\int_{\mathbb{R}^{d}} g(y) \delta_{Y}(y) d y=g(Y) \quad \text { a.s. }
$$

for all (measurable) $g: \mathbb{R}^{d} \rightarrow \mathbb{R}$ such that the integral converges in $(\mathcal{S})_{H}^{*}$.

As in [AØU, Prop. 4.2] we can now prove

Proposition 4.2 Suppose $Y: \Omega \rightarrow \mathbb{R}^{d}$ is normally distributed with mean $m=\mathbb{E}_{\mu^{(H)}}[Y]$ and covariance matrix $C=\left[c_{j k}\right]_{1 \leq j, k \leq d}$. Suppose $C$ is invertible with inverse

$$
A=C^{-1}=\left[a_{j k}\right]_{1 \leq j, k \leq d} .
$$

Then $\delta_{Y}(y)$ exists, is unique and given by

$$
\delta_{Y}(y)=(2 \pi)^{-d / 2} \sqrt{|A|} \exp ^{\diamond}\left[-\frac{1}{2} \sum_{j, k=1}^{d} a_{j k}\left(Y_{j}+m_{j}-y_{j}\right) \diamond\left(Y_{k}+m_{k}-y_{k}\right)\right]
$$

where $|A|$ is the determinant of $A$. 
Proof The proof of Proposition 4.2 in [AØU] applies, if we replace the standard Hida distribution space $(\mathcal{S})^{*}$ and its corresponding (standard) Wick product by the fractional distribution space $(\mathcal{S})_{H}^{*}$ and the fractional Wick product. We omit the details.

Proposition 4.3 Let $Y, C=\left[c_{j k}\right], A=C^{-1}=\left[a_{j k}\right]$ be as in Proposition 2.2. Then

$$
\delta_{Y}(y)=(2 \pi)^{-d} \int_{\mathbb{R}^{d}} \exp ^{\diamond}\left[i \sum_{j=1}^{d} \xi_{j}\left(Y_{j}+m_{j}-y_{j}\right)-\frac{1}{2} \sum_{j, k=1}^{d} c_{j k} \xi_{j} \xi_{k}\right] d \xi
$$

where $i=\sqrt{-1}$ is the imaginary unit.

Proof Recall the well-known formula

$$
\int_{\mathbb{R}^{d}} \exp \left[b^{T} \xi-\frac{1}{2} \xi^{T} C \xi\right] d \xi=(2 \pi)^{d / 2}|A|^{1 / 2} \exp \left[\frac{1}{2} b^{T} A b\right]
$$

valid for all $b \in \mathbb{C}^{d}$.

Using the fractional Hermite transform (see [HØUZ] for the standard case), we obtain the following Wick analogue of (4.10)

$$
\int_{\mathbb{R}^{d}} \exp ^{\diamond}\left[b^{T} \xi-\frac{1}{2} \xi^{T} C \xi\right] d \xi=(2 \pi)^{d / 2}|A|^{1 / 2} \exp ^{\diamond}\left[\frac{1}{2} b^{T} \diamond A \diamond b\right],
$$

valid for all $b \in L^{2}\left(\mu^{(H)}\right)$. In particular, if we apply (4.10) with $b=i(Y+m-y)$ we get from (4.8) that

$$
\delta_{Y}(y)=(2 \pi)^{-d / 2}|A|^{1 / 2} \exp ^{\diamond}\left[\frac{1}{2} b^{T} \diamond A \diamond b\right]=(2 \pi)^{-d} \int_{\mathbb{R}^{d}} \exp ^{\diamond}\left[b^{T} \xi-\frac{1}{2} \xi^{T} C \xi\right] d \xi
$$

which is (4.9).

From this we deduce the Hu formula for the Donsker delta function:

Theorem 4.4 ([Hu1])

$$
\delta_{Y}(y)=(2 \pi)^{-d} \int_{\mathbb{R}^{d}} \exp \left(i \sum_{j=1}^{N} \xi_{j}\left(Y_{j}+m_{j}-y_{j}\right)\right) d \xi
$$

where the integral exists in $(\mathcal{S})_{H}^{*}$. 
Proof Recall the following connection between the Wick exponential and the ordinary exponential of a Gaussian random variable $Z$ with values in $\mathbb{R}^{d}$ and with $\mathbb{E}[Z]=0$ : (See $[\mathrm{H} \varnothing,(3.35)]$ for a proof)

$$
\exp ^{\diamond}\left(b^{T} Z\right)=\exp \left(b^{T} Z-\frac{1}{2} b^{T} \mathbb{E}\left[Z Z^{T}\right] b\right)
$$

for all (deterministic) $b \in \mathbb{C}^{d}$.

If we apply this to (4.9) with $Z=Y-m$ and $b=i \xi$ we get (4.12).

We now specialize to the case when $Y=B^{(H)}(t)$ :

Corollary 4.5 The Donsker delta function $\delta_{B^{(H)}(t)}(x)$ of fractional Brownian $B^{(H)}(t) \in \mathbb{R}^{d}$ is given by

$$
\delta_{B^{(H)}(t)}(x)=(2 \pi)^{-d / 2}\left(\prod_{j=1}^{d} t^{-H_{j}}\right) \exp ^{\diamond}\left[-\frac{1}{2} \sum_{j=1}^{d} t^{-2 H_{j}}\left(B_{j}^{(H)}(t)-x_{j}\right)^{\circ 2}\right] .
$$

Proof The random variable $Y:=B^{(H)}(t)$ is normally distributed with mean 0 and with covariance matrix $C=\left[c_{j k}\right]$ given by (see (4.4))

$$
c_{j k}=\mathbb{E}_{\mu_{H}}\left[B_{j}^{(H)}(t) B_{k}^{(H)}(t)\right]=t^{2 H_{j}} \delta_{j k} ; \quad 1 \leq j, k \leq d .
$$

Thus $C$ is diagonal with inverse $A=\left[a_{j k}\right]$ given by

$$
a_{j k}=t^{-2 H_{j}} \delta_{j k} .
$$

Then

$$
|A|=\prod_{j=1}^{d} t^{-2 H_{j}} .
$$

Hence the result follows from Proposition 2.2.

Corollary 4.6 The Donsker delta function is also given by

$$
\begin{aligned}
& \delta_{B^{(H)}(t)}(x) \\
& =(2 \pi)^{-d} \int_{\mathbb{R}^{d}} \exp ^{\diamond}\left[i \sum_{j=1}^{d} \xi_{j}\left(B_{j}^{(H)}(t)-x_{j}\right)-\frac{1}{2} \sum_{j=1}^{d} t^{2 H_{j}} \xi_{j}^{2}\left(B_{j}^{(H)}(t)-x_{j}\right)^{\diamond 2}\right] d \xi .
\end{aligned}
$$

Proof This follows from Proposition 2.3 and (4.15). 
Remark. Note that this integral converges in the fractional distribution space $(\mathcal{S})_{H}^{*}$. This follows by considering the fractional Hermite transforms. See [HØUZ].

\section{Corollary 4.7}

$$
\delta_{B^{(H)}(t)}(x)=(2 \pi)^{-d} \int_{\mathbb{R}^{d}} \exp \left[i \sum_{j=1}^{d} \xi_{j}\left(B^{(H)}(t)-x_{j}\right)\right] d \xi
$$

where the integral converges in $(\mathcal{S})_{H}^{*}$.

Proof This is a direct consequence of Theorem 2.4.

We now proceed to define fractional local time:

Definition 4.8 Fix $T>0$ and a point $x \in \mathbb{R}^{d}$. The local time of $B^{(H)}(t)$ up to time $T$ at the point $x$ is defined by

$$
\ell_{T}^{(H)}(x)=\lim _{\varepsilon \rightarrow 0} \frac{1}{|D(x, \varepsilon)|} \lambda\left(\left\{s \in[0, T] ; B^{(H)}(s) \in D(x, \varepsilon)\right\}\right)
$$

$\left(\operatorname{limit}\right.$ in $\left.(\mathcal{S})_{H}^{*}\right)$, where

$$
D(x, \varepsilon)=\left\{y \in \mathbb{R}^{d} ;|y-x|<\varepsilon\right\},
$$

$|D(x, \varepsilon)|=\lambda(D(x, \varepsilon))$ is the volume of $D(x, \varepsilon)$ and $\lambda$ denotes the Lebesgue measure in $\mathbb{R}^{d}$.

Remark. This definition is natural from the point of view that local time at $x$ is the amount of time spent at the point.

Proposition 4.9 a) The local time of $B^{(H)}(t) \in \mathbb{R}^{d}$ at the point $x \in \mathbb{R}^{d}$ exists in $(\mathcal{S})_{H}^{*}$ and is given by

$$
\ell_{T}^{(H)}(x)=\int_{0}^{T} \delta_{B^{(H)}(s)}(x) d s
$$

b) Hence, for d-dimensional $B^{(H)}(t)$ we have

$$
\ell_{T}^{(H)}(x)=(2 \pi)^{-d / 2} \int_{0}^{T}\left(\prod_{j=1}^{d} t^{-H_{j}}\right) \exp ^{\diamond}\left[-\frac{1}{2} \sum_{j=1}^{d} t^{-2 H_{j}}\left(B_{j}^{(H)}(t)-x_{j}\right)^{\diamond 2}\right] d t
$$

and

$$
\ell_{T}^{(H)}(x)=(2 \pi)^{-d} \int_{0}^{T}\left(\int_{\mathbb{R}^{d}} \exp \left[i \sum_{j=1}^{d} \xi_{j}\left(B^{(H)}(t)-x_{j}\right)\right] d \xi\right) d t
$$


c) In particular, for $d=1$ this gives

$$
\ell_{T}^{(H)}(x)=(2 \pi)^{-1 / 2} \int_{0}^{T} t^{-H} \exp ^{\diamond}\left[-\frac{1}{2} t^{-H}\left(B^{(H)}(t)-x\right)^{\diamond 2}\right] d t
$$

and

$$
\ell_{T}^{(H)}(x)=(2 \pi)^{-1} \int_{0}^{T}\left(\int_{\mathbb{R}} \exp \left[i \xi\left(B^{(H)}(t)-x\right)\right] d \xi\right) d t .
$$

Proof If we apply (4.7) to the function

$$
g_{\varepsilon}(y)=\chi_{D(x, \varepsilon)}(y)= \begin{cases}1 & \text { if } y \in D(x, \varepsilon) \\ 0 & \text { otherwise }\end{cases}
$$

we get

$$
\begin{aligned}
\lambda(\{s & \left.\left.\in[0, T] ; B^{(H)}(s) \in D(x, \varepsilon)\right\}\right) \\
& =\int_{0}^{T} \chi_{D(x, \varepsilon)}\left(B^{(H)}(s)\right) d s \\
& =\int_{0}^{T}\left(\int_{\mathbb{R}^{d}} \chi_{D(x, \varepsilon)}(y) \delta_{B^{(H)}(s)}(y) d y\right) d s \\
& =\int_{D(x, \varepsilon)}\left(\int_{0}^{T} \delta_{B^{(H)}(s)}(y) d s\right) d y .
\end{aligned}
$$

Hence

$$
\begin{aligned}
& \ell_{T}^{(H)}(x)=\lim _{\varepsilon \rightarrow 0} \frac{1}{|D(x, \varepsilon)|} \lambda\left(\left\{s \in[0, T] ; B^{(H)}(s) \in D(x, \varepsilon)\right\}\right) \\
& =\lim _{\varepsilon \rightarrow 0} \frac{1}{|D(x, \varepsilon)|} \int_{D(x, \varepsilon)}\left(\int_{0}^{T} \delta_{B^{(H)}(s)}(y) d s\right) d y=\int_{0}^{T} \delta_{B^{(H)}(s)}(x) d s .
\end{aligned}
$$

Formulas (4.22) and (4.23) now follow from Corollary 2.5 and Corollary 2.7.

The generalized expectation operator $\mathbb{E}$ can be defined on $(\mathcal{S})_{H}^{*}$ in exactly the same way as in the standard case (see [HØUZ]): If $X \in(\mathcal{S})_{H}^{*}$ then $\mathbb{E}[X]$ is the zero order element in the chaos expansion of $X$. In particular, $\mathbb{E}$ has the properties

$$
\mathbb{E}[Y \diamond Z]=\mathbb{E}[Y] \mathbb{E}[Z] \quad \text { for } Y, Z \in(\mathcal{S})_{H}^{*}
$$

and

$$
\mathbb{E}\left[\exp ^{\diamond} Y\right]=\exp \mathbb{E}[Y] \quad \text { if } \exp ^{\diamond} Y \in(\mathcal{S})_{H}^{*}
$$

Therefore we obtain the following directly from (4.22) and (4.24): 
Corollary 4.10 a) The generalized expectation of fractional local time of $B^{(H)}(t) ; 0 \leq t \leq$ $T$ at $x \in \mathbb{R}^{d}$ is

$$
\mathbb{E}\left[\ell_{T}^{(H)}(x)\right]=(2 \pi)^{-d / 2} \int_{0}^{T}\left(\prod_{j=1}^{d} t^{-H_{j}}\right) \exp \left(-\frac{1}{2} \sum_{j=1}^{d} t^{-2 H_{j}} x_{j}^{2}\right) d t .
$$

b) In particular, for $d=1$ we have

$$
\mathbb{E}\left[\ell_{T}^{(H)}(x)\right]=(2 \pi)^{-1 / 2} \int_{0}^{T} t^{-H} \exp \left(-\frac{1}{2} t^{-2 H} x^{2}\right) d t .
$$

In the next section we find the whole chaos expansion of $\ell_{T}^{(H)}(x)$. Then we will find conditions which ensure that $\ell_{T}^{(H)}(x, \cdot) \in L^{2}\left(\mu^{(H)}\right)$. If this is the case then (4.28), (4.29) give the usual expectation of $\ell_{T}^{(H)}(x, \cdot)$.

It is well-known that $L^{2}\left(\mu^{(H)}\right)$ is a dense subset of $(\mathcal{S})_{H}^{*}$. Thus $\ell_{T}^{(H)}(x)$ can be approximated by elements in $L^{2}\left(\mu^{(H)}\right)$. It is easy to verify that

Proposition 4.11 Let $P_{\varepsilon}$ be defined as in (1.3) and let

$$
\ell_{\varepsilon, T}^{(H)}(x)=\int_{0}^{T} P_{\varepsilon}\left(B^{(H)}(t)-x\right) d t=\frac{1}{(2 \pi)^{d}} \int_{0}^{T} \int_{\mathbb{R}^{d}} e^{i\left(B^{(H)}(t)-x\right) \xi-\frac{1}{2} \xi^{2}} d \xi d t
$$

Then

$$
\lim _{\varepsilon \rightarrow 0} \ell_{\varepsilon, T}^{(H)}(x)=\ell_{T}^{(H)}(x) \quad \text { in } \quad(\mathcal{S})_{H}^{*}
$$

\section{The chaos expansion of fractional local time}

In this section we will use the following formula for the Donsker delta function,

$$
\delta_{B^{(H)}(t)}(x)=(2 \pi)^{-d} \int_{\mathbb{R}^{d}} \exp \left[i \sum_{j=1}^{d} \xi_{j}\left(B^{(H)}(t)-x_{j}\right)\right] d \xi \in(\mathcal{S})_{H}^{*}
$$

obtained in Corollary 4.7, to find the chaos expansion of the fractional local time

$$
\ell_{T}^{(H)}(x)=\int_{0}^{T} \delta_{B^{(H)}(t)}(x) d t
$$

For simplicity we will first assume that $x=0$ (the case $x \neq 0$ is similar) and we will put

$$
\delta\left(B^{(H)}(t)\right)=\delta_{B^{(H)}(t)}(0)
$$


Let $f(s)=\left(f_{1}(s), \cdots, f_{d}(s)\right)$ be a (complex) deterministic function, $f=0$ outside $[0, T]$. Define

$$
\mathcal{E}[f](t):=\exp \left(\int_{0}^{t} f(s) d B^{(H)}(s)-\frac{1}{2}\left\|f \chi_{[0, t]}\right\|_{H}^{2}\right) .
$$

Then it is easy to see that

$$
\mathcal{E}[f](t)=1+\sum_{j=1}^{d} \int_{0}^{t} \mathcal{E}[f](s) f_{j}(s) d B_{j}^{(H)}(s) .
$$

By iteration of this identity it follows that

$$
\begin{aligned}
& \exp \left(\int_{0}^{T} f(s) d B^{(H)}(s)-\frac{1}{2}\|f\|_{\varphi}^{2}\right) \\
& \quad=1+\sum_{n=1}^{\infty} \sum_{1 \leq j_{1}, \cdots, j_{n} \leq d} \int_{0<s_{1}<\cdots<s_{n}<T} f_{j_{1}}\left(s_{1}\right) \cdots f_{j_{n}}\left(s_{n}\right) d B_{j_{1}}^{(H)}\left(s_{1}\right) \cdots d B_{j_{n}}^{(H)}\left(s_{n}\right) .
\end{aligned}
$$

Note that

$$
i B^{(H)}(t) \xi=i \sum_{j=1}^{d} \xi_{j} \int_{0}^{T} \chi_{[0, t]}(s) d B_{j}^{(H)}(s) \quad \text { for all } \quad \xi \in \mathbb{R}^{d}
$$

and

$$
\chi_{[0, t]}\left(s_{1}\right) \ldots \chi_{[0, t]}\left(s_{n}\right)=\chi_{\left[\max \left\{s_{1}, \cdots, s_{n}\right\}, \infty\right)}(t) .
$$

Thus for any $\xi \in \mathbb{R}^{d}$,

$$
\begin{aligned}
\exp & \left(i \xi B^{(H)}(t)\right)=\exp \left(i \xi B^{(H)}(t)+\frac{1}{2} \sum_{j=1}^{d} t^{2 H_{j}} \xi_{j}^{2}\right) \exp \left(-\frac{1}{2} \sum_{j=1}^{d} t^{2 H_{j}} \xi_{j}^{2}\right) \\
= & \sum_{n=0}^{\infty} i^{n} \exp \left(-\frac{1}{2} \sum_{j=1}^{d} t^{2 H_{j}} \xi_{j}^{2}\right) \\
& \cdot \int_{0<s_{1}<\cdots<s_{n}<T} \sum_{1 \leq j_{1}, \ldots, j_{n} \leq d} \xi_{j_{1}} \ldots \xi_{j_{n}} \chi_{[0, t]}\left(s_{1}\right) \ldots \chi_{[0, t]}\left(s_{n}\right) d B_{j_{1}}^{(H)}\left(s_{1}\right) \ldots d B_{j_{n}}^{(H)}\left(s_{n}\right) \\
= & \sum_{n=0}^{\infty} i^{n} \exp \left(-\frac{1}{2} \sum_{j=1}^{d} t^{2 H_{j}} \xi_{j}^{2}\right) \\
& \cdot \sum_{0<s_{1}<\cdots<s_{n}<T} \sum_{1 \leq j_{1}, \ldots, j_{n} \leq d} \xi_{j_{1}} \ldots \xi_{j_{n}} \chi_{\left[\max \left\{s_{1}, \ldots, s_{n}\right\}, \infty\right)}(t) d B_{j_{1}}^{(H)}\left(s_{1}\right) \ldots d B_{j_{n}}^{(H)}\left(s_{n}\right)
\end{aligned}
$$

Therefore by Proposition 4.9 b)

$$
\ell_{T}^{(H)}(0)=\sum_{n=0}^{\infty} i^{n} \sum_{1 \leq j_{1}, \ldots, j_{n} \leq d} \int_{0<s_{1}<\cdots<s_{n}<T} f_{j_{1}, \ldots, j_{n}}\left(s_{1}, \ldots, s_{n}\right) d B_{j_{1}}^{(H)}\left(s_{1}\right) \cdots d B_{j_{n}}^{(H)}\left(s_{n}\right)
$$


where, for $n \geq 1$,

$$
\begin{aligned}
& f_{j_{1}, \ldots, j_{n}}\left(s_{1}, \ldots, s_{n}\right) \\
& \quad=\frac{1}{(2 \pi)^{d}} \int_{0}^{T} \int_{\mathbb{R}^{d}} \exp \left(-\frac{1}{2} \sum_{k=1}^{d} t^{2 H_{k}} \xi_{k}^{2}\right) \xi_{j_{1}} \ldots \xi_{j_{n}} \chi_{\left[\max \left\{s_{1}, \ldots, s_{n}\right\}, \infty\right)}(t) d t d \xi_{1} \cdots d \xi_{d} .
\end{aligned}
$$

To compute $f_{j_{1}, \ldots, j_{n}}\left(s_{1}, \ldots, s_{n}\right)$, let us introduce

$$
j=\left(j_{1}, j_{2}, \ldots, j_{n}\right)
$$

and

$$
\nu(j, k):=\#\left\{l \in\{1,2, \ldots, n\} ; j_{l}=k\right\}
$$

for $k=1,2, \ldots, d$. Thus $\nu(j, 1)+\cdots+\nu(j, d)=n$ for $j=1, \cdots, n$. With this notation, we obtain

$$
\begin{aligned}
\int_{\mathbb{R}^{d}} \exp & \left(-\frac{1}{2} \sum_{k=1}^{d} t^{2 H_{k}} \xi_{k}^{2}\right) \xi_{j_{1}} \cdots \xi_{j_{n}} d \xi_{1} \cdots d \xi_{d} \\
& =\int_{\mathbb{R}^{d}} \exp \left(-\frac{1}{2} \sum_{k=1}^{d} t^{2 H_{k}} \xi_{k}^{2}\right) \xi_{1}^{\nu(j, 1)} \cdots \xi_{d}^{\nu(j, d)} d \xi_{1} \cdots d \xi_{d} \\
& =t^{-K(j)} \int_{\mathbb{R}^{d}} e^{-\frac{1}{2}|\eta|^{2}} \eta_{1}^{\nu(j, 1)} \cdots \eta_{d}^{\nu(j, d)} d \eta_{1} \cdots d \eta_{d} \\
& =t^{-K(j)}(2 \pi)^{d} C(j)
\end{aligned}
$$

where

$$
C(j)= \begin{cases}0 & \text { when one of } \nu(j, k) \text { is odd } \\ (2 \pi)^{-d / 2} \frac{\nu(j, k) !}{2^{\nu(j, k) / 2}(\nu(j, k) / 2) !} & \text { when all } \nu(j, k) \text { are even }\end{cases}
$$

and

$$
K(j)=\sum_{k=1}^{d} H_{k}(1+\nu(j, k))
$$

Thus

$$
\begin{aligned}
& f_{j_{1}, \ldots, j_{n}}\left(s_{1}, \ldots, s_{n}\right)=C(j) \int_{\max \left\{s_{1}, \ldots, s_{n}\right\}}^{T} t^{-K(j)} d t \\
& =\frac{C(j)}{1-K(j)}\left(T^{1-K(j)}-\max \left\{s_{1}, \ldots, s_{n}\right\}^{1-K(j)}\right) .
\end{aligned}
$$

Consequently

$$
\ell_{T}^{(H)}(0)=\sum_{n \text { even }} \sum_{1 \leq j_{1}, \ldots, j_{n} \leq d} \int_{0<s_{1}<\cdots<s_{n}<T} g_{n}\left(T, \max \left\{s_{1}, \ldots, s_{n}\right\}\right) d B_{j_{1}}^{(H)}\left(s_{1}\right) \cdots d B_{j_{n}}^{(H)}\left(s_{n}\right),
$$


where

$$
g_{n}(T, u)=(-1)^{n} \frac{C(j)}{1-K(j)}\left(T^{1-K(j)}-u^{1-K(j)}\right) \quad \text { for } \quad n \geq 1 .
$$

(When $n=0$, we assume that $\max \left\{s_{1}, \ldots, s_{n}\right\}=0$.) Thus we obtain

Theorem 5.1 The chaos expansion of the fractional local time $\ell_{T}^{(H)}(0)=\int_{0}^{T} \delta(B(t)) d t$ at $x=0$ is given by

$$
\begin{aligned}
\ell_{T}^{(H)}(0) & =\int_{0}^{T} \delta(B(t)) d t \\
& =\sum_{n \text { even }} \sum_{1 \leq j_{1}, \ldots, j_{n} \leq d} \int_{0<s_{1}<\cdots<s_{n}<T} g_{n}\left(T, \max \left\{s_{1}, \ldots, s_{n}\right\}\right) d B_{j_{1}}^{(H)}\left(s_{1}\right) \ldots d B_{j_{n}}^{(H)}\left(s_{n}\right),
\end{aligned}
$$

where $g(T, u)$ is given by (5.8).

Remark 5.2 If the series in (5.9) converges in $L^{2}(\mu)$ then the expectation is the zero order term in the chaos expansion. Thus by choosing $n=0$ in (5.8) we have, for $d=1$, (see Corollary 4.10)

$$
\mathbb{E}\left(\int_{0}^{T} \delta(B(t)) d t\right)=\frac{T^{1-H}}{\sqrt{2 \pi}(1-H)}
$$

Now we compute the $L^{2}$ norm of the local time of the fractional Brownian motions. We still use the expression (5.1)-(5.2) for the Donsker delta function. It suffices to show that $\ell_{\varepsilon, T}^{(H)}(0)$ is a bounded sequence in $L^{2}\left(\mu^{(H)}\right)$ as $\varepsilon \rightarrow 0+$. To simplify the writing, we let $\varepsilon=0$. Thus we need to estimate

$$
\begin{aligned}
\mathbb{E}\left\{\left[\ell_{T}^{(H)}(x)\right]^{2}\right\} & =\mathbb{E}\left[\int_{0}^{T} \delta\left(B^{(H)}(t)-x\right) d t\right]^{2} \\
& =\frac{1}{(2 \pi)^{2 d}} \int_{[0, T]^{2}} \int_{\mathbb{R}^{2 d}} e^{-i \xi x+i \eta x} \mathbb{E} e^{-i \xi B^{(H)}(t)+i \eta B^{(H)}(s)} d \xi d \eta d s d t \\
& \leq \frac{1}{(2 \pi)^{2 d}} \int_{[0, T]^{2}} \int_{\mathbb{R}^{2 d}} e^{-\frac{1}{2} \operatorname{Var}\left(\xi B^{(H)}(t)-\eta B^{(H)}(s)\right)} d \xi d \eta d s d t \\
& \leq \frac{2}{(2 \pi)^{2 d}} \int_{0 \leq s<t \leq T} \int_{\mathbb{R}^{2 d}} e^{-\frac{1}{2} \operatorname{Var}\left(\xi B^{(H)}(t)-\eta B^{(H)}(s)\right)} d \xi d \eta d s d t
\end{aligned}
$$

since for any Gaussian random variable $X$,

$$
\mathbb{E} e^{i X}=e^{-\frac{1}{2} \operatorname{Var}(X)} .
$$


Using the nondeterminism property of fractional Brownian motions we have that when $0 \leq$ $s<t \leq T$, there is a positive constant $k>0$ such that

$$
\begin{aligned}
\operatorname{Var}\left(\xi B^{(H)}(t)-\eta B^{(H)}(s)\right) & =\operatorname{Var}\left(\xi\left(B^{(H)}(t)-B^{(H)}(s)\right)+(\xi-\eta) B^{(H)}(s)\right) \\
& \geq k\left[\xi^{2}|t-s|^{2 H_{0}}+(\eta-\xi)^{2} s^{2 H_{0}}\right]
\end{aligned}
$$

where

$$
H_{0}=\max \left(H_{1}, \ldots, H_{d}\right) .
$$

See e.g. [Be1], [Be2], [Be3], [Be4], [Be5], [Hu3], [Ro] for the use of this property.

Therefore, we have when $H_{0} d<1$,

$$
\begin{aligned}
\mathbb{E}\left\{\left[\ell_{T}^{(H)}(x)\right]^{2}\right\} & \leq \frac{2}{(2 \pi)^{2 d}} \int_{0 \leq s<t \leq T} \int_{\mathbb{R}^{2 d}} e^{-\frac{k}{2}\left(\xi^{2}|t-s|^{2 H_{0}}+(\eta-\xi)^{2} s^{2 H_{0}}\right)} d \xi d \eta d s d t \\
& =\frac{2}{(2 \pi)^{d} k^{d / 2}} \int_{0 \leq s<t \leq T} \frac{1}{s^{H_{0} d}(t-s)^{H_{0} d}} d s d t \\
& =\frac{2}{(2 \pi)^{d} k^{d / 2}} \int_{0}^{T}\left(\int_{0}^{t} \frac{1}{s^{H_{0} d}(t-s)^{H_{0} d}} d s\right) d t \\
& =\frac{2 \Gamma\left(1-H_{0} d\right)^{2}}{(2 \pi)^{d} k^{d / 2} \Gamma\left(2-2 H_{0} d\right)} \int_{0}^{T} t^{1-2 H_{0} d} d t \\
& =\frac{2 \Gamma\left(1-H_{0} d\right)^{2}}{(2 \pi)^{d} k^{d / 2} \Gamma\left(2-2 H_{0} d\right)\left(2-2 H_{0} d\right)} T^{2-2 H_{0} d} \\
& =\frac{2 \Gamma\left(1-H_{0} d\right)^{2}}{(2 \pi)^{d} k^{d / 2} \Gamma\left(3-2 H_{0} d\right)} T^{2-2 H_{0} d} .
\end{aligned}
$$

Summarizing the above, we obtain

Theorem 5.3 Assume that $H_{0} d<1$. Then the local time $\ell_{T}^{(H)}(x)=\int_{0}^{T} \delta(B(t)-x) d t$ is square integrable and for any $x \in \mathbb{R}$ we have

$$
\mathbb{E}\left[\int_{0}^{T} \delta(B(t)-x) d t\right]^{2} \leq \frac{2 \Gamma\left(1-H_{0} d\right)^{2} T^{2-2 H_{0} d}}{(2 \pi) \sqrt{k} \Gamma\left(3-2 H_{0} d\right)} .
$$

where $k$ is a constant depending on $H_{0}$ and is defined by (5.11).

Acknowledgments. Hu is partly supported by the National Science Foundation under Grant No. EPS-9874732, matching support from the State of Kansas and General Research Fund of the University of Kansas. 


\section{References}

[AMN] E. Alòs, E. Mazet and D. Nualart: Stochastic calculus with respect to fractional Brownian motion with Hurst parameter less than 1/2. Stochastic Process. Appl. 86 (2000), no. 1, 121-139.

[AHZ] S. Albeverio, Y. Hu, and X.Y. Zhou: A remark on non smoothness of self intersection local time of planar Brownian motion. Statist. Probab. Letter, 32 (1997), 57-65.

[AØU] K. Aase, B. Øksendal and J. Ubøe: Using the Donsker delta function to compute hedging strategies. To appear in Potential Analysis.

[Be1] S.M. Berman: A central limit theorem for the renormalized self-intersection local time of a stationary process. Probability in Banach spaces, 8 (Brunswick, ME, 1991), 351363, Progr. Probab., 30, Birkhäuser Boston, Boston, MA, 1992.

[Be2] S.M. Berman: Self-intersections and local nondeterminism of Gaussian processes. Ann. Probab. 19 (1991), no. 1, 160-191.

[Be3] S.M. Berman: Local nondeterminism and local times of general stochastic processes. Ann. Inst. H. Poincaré Sect. B (N.S.) 19 (1983), no. 2, 189-207.

[Be4] S. M. Berman: Local nondeterminism and local times of Gaussian processes. Indiana Univ. Math. J. 23 (1973/74), 69-94.

[Be5] S.M. Berman: Local nondeterminism and local times of Gaussian processes. Bull. Amer. Math. Soc. 79 (1973), 475-477.

[DHP] T. E. Duncan, Y. Hu and B. Pasik-Duncan: Stochastic calculus for fractional Brownian motion. I. Theory. SIAM J. Control Optim. 38 (2000), 582-612.

[HKPS] T. Hida, H.-H Kuo, J. Potthoff and L. Streit: White Noise Analysis. Kluwer, 1993.

[Hu1] Y. Hu: On the self-intersection local time of Brownian motion - via chaos expansion, Publicacions Matemátiques, vol. 40 (1996), 337-350.

[Hu2] Y. Hu: Itô-Wiener chaos expansion with exact residual and correlation, variance inequalities. Journal of Theoretical Probability, 10 (1997), 835-848.

[Hu3] Y. Hu: Self-intersection local time of fractional Brownian motions - via chaos expansion. Preprint. 
[Hu4] Y. Hu: Prediction and translation of fractional Brownian motions. In T. Hida et al (editors): Stochastics in finite and infinite dimensions, 153-171, Trends Math., Birkhäuser Boston, Boston, MA, 2001.

[HØ] Y.Hu and B. Øksendal: Fractional white noise calculus and application to finance. Preprint 10/1999, University of Oslo.

[HØUZ] H. Holden, B. Øksendal, J. Ubøe and T. Zhang: Stochastic Partial Differential Equations. Birkhäuser 1996.

[HØZ] Y. Hu, B. Øksendal and T. Zhang: Stochastic partial differential equations driven by multiparameter fractional white noise. In F. Gesztesy et al. (editors): Stochastic Processes, Physics and Geometry: New Interplays, II (Leipzig, 1999), 317-325, CMS Conf. Proc., 29, Amer. Math. Soc., Providence, RI, 2000.

[J] S. Janson: Gaussian Hilbert spaces. Cambridge Tracts in Mathematics, 129. Cambridge University Press, Cambridge, 1997.

[LØU] T. Lindstrøm, B. Øksendal and J. Ubøe: Wick multiplication and Itô-Skorohod stochastic differential equations. in S. Albeverio, J.E. Fenstad, H. Holden and T. Lindstrøm (editors): Ideas and Methods in Mathematical Analysis, Stochastics, and Applications, Cambridge Univ. Press, 1992, pp. 183-206

[MvN] B.B. Mandelbrot and J.W. van Ness: Fractional Brownian motion, fractional noises and applications. SIAM Rev. 10 (1968), 422-437.

[Ne] J. Neveu: Processus Aléatoires Gaussiens. Les Presses de l'Université de Montréal, Montreal, Que. 1968.

[NV] D. Nualart and J. Vives: Chaos expansion and local time, Publications Matematiques $36(2), 1992,827-836$.

[NVV] I. Norros, E. Valkeila and J. Virtamo: An elementary approach to a Girsanov formula and other analytic results on fractional Brownian motions. Bernoulli 5 (1999), 571-587.

[ØZ] B. Øksendal and T. Zhang: Multiparameter fractional Brownian motion and quasilinear stochastic partial differential equations. To appear in Stochastics and Stochastics Reports.

[Ro] J. Rosen: The intersection local time of fractional Brownian motion in the plane. J. Multivariate Anal. 23 (1987), no. 1, 37-46. 
[SKM] S. G. Samko, A. A. Kilbas and O. I. Marichev: Fractional Integrals and Derivatives, Theory and Applications, Gordon and Breach Science Publishers, 1993.

[Wid] H. Widom: Stable processes and integral equations. Trans. Amer. Math. Soc. 98 (1961), 430-449. 